\title{
EFFECT OF HIGH ELECTROSTATIC ACTUATION ON THERMOELASTIC DAMPING IN THIN RECTANGULAR MICROPLATE RESONATORS
}

\author{
Ardeshir Karami Mohammadi, Nassim Ale Ali \\ Department of Mechanical Engineering, Shahrood University of Technology, Iran \\ e-mail: akaramim@yahoo.com
}

\begin{abstract}
In this paper, a micro resonator is modeled as a thin rectangular microplate with thermoelastic damping that is actuated electrostatically. Large static deformation due to high polarization voltage is considered, and vibration of microplate occurs around the static deflection. Due to the effect of thermoelastic damping, the frequency of vibration is a complex value that is used to determine the quality factor of thermoelastic damping. Also, the pull-in voltage is considered because nonlinear properties are more appeared when approaching the polarization voltage to the pull-in voltage.
\end{abstract}

Keywords: microplate resonator, thermoelastic damping, high polarization voltage

\section{Introduction}

Thermoelastic damping is the intrinsic damping in Micro Electro Mechanical Systems (MEMS). It arises from the entropy generation due to irreversible heat flux in vibrating device (Sun and Saka, 2009). Zener $(1937,1938)$ was the first who predicted thermo-elastic damping. He found an expression for quality factor of thermoelastic damping in beams. Conversion of mechanical energy into heat in vibrating elastic beams was treated by Alblas (1981). He also found that this damping is negligible for macro structures. Lifshitz and Roukes (2000) investigated the quality factor of a thermo-elastic microbeam and found that thermoelastic damping has important effects in micro and nano scales.

There are two methods for calculating the quality factor of thermoelastic damping: energy method, in which dissipated and maximum stored energy should be calculated (Sudipto et al., 2006; Prabhakar and Vengallatore, 2008; Serra and Bonaldi, 2009; Guo and Rogerson, 2003), and eigenfrequency method, in which real and imaginary parts of the eigenfrequency should be calculated (Sun and Saka, 2009; Nayfeh and Younis, 2004b; Yi and Matin, 2007; Choi et al., 2010). Each method can be done with numerical or analytical procedures or combination of them.

Nayfeh and Younis (2004b) modeled the electrostatically actuated microplate by considering thermoelastic damping. They obtained an expression for quality factor analytically by using perturbation theory. Sudipto and Aluru (2006) investigated thermoelastic damping in an electrostatically actuated microbeam by the thermal energy method. They studied the effect of applied voltage on thermoelastic damping.

In MEMS, there are different actuation and sensing properties such as thermal, optical, electrostatic, electromagnetic, piezoresistive and piezoelectric but electrostatics is often preferred (Fargas Marquès et al., 2005). In electrostatics actuation, an elastic conductor is located above a stationary conductor. The electrical load can be composed of two components, including DC and AC voltage. The applied DC voltage deforms the elastic surface that causes to change the system capacitance and to stretch the mid-plane of the elastic surface. The applications are transistors, switches, micro-mirrors, pressure sensors, micro-pumps, moving valves and micro-grippers which 
have no harmonic motion in their systems. If AC voltage is added then resonators are obtained (Batra et al., 2007).

There are many works and papers that investigated the electrical actuation in micro structures. Batra et al. (2007) reviewed them in their work. Abdel-Rahman et al. (2002) presented a nonlinear model of electrically actuated microbeams considering mid-plane stretching. They showed static deflection of the microbeam due to DC polarization and vibration of the microbeam around its statically deflected position.

MEMS resonators are devices that vibrate with $\mathrm{AC}$ voltage around the static deflection due to DC polarization voltage. The thermoelastic damping as well as frequency of the structure of the resonator is affected by the $\mathrm{DC}$ voltage because the thermoelastic damping is directly related to the imaginary part of the frequency. In addition, in MEMS resonators, high sensitivity and resolution are needed (Nayfeh and Younis, 2004a), so for achieving this purpose, the damping in such devices should be decreased. However, studying the behavior of thermoelastic damping in resonators is necessary for manufacturers of MEMS.

In this paper, a resonator is modeled as a thin rectangular microplate with thermoelastic damping that is actuated electrostatically. Large static deformation due to high polarization voltage is considered, and vibration of the microplate occurs around the static deflection. Because of thermoelastic damping, the frequency of vibration is a complex value that is used to determine the quality factor of thermoelastic damping. Also, the pull-in voltage is investigated because nonlinear properties are more appeared when approaching the polarization voltage to the pullin voltage.

\section{Model description and assumptions}

A resonator is modeled as a rectangular microplate subject to the effect of a high electrostatic polarization voltage $V_{p}$. The equations of motion of the isotropic thin microplate are derived by using the combination of the classical plate theory and von-Karman type nonlinearity (Nayfeh and Pai, 2004). A Cartesian coordinate system $(x, y, z)$ is attached to the microplate such that the $x y$ plane corresponds to the mid-plane of the rectangular microplate over the domain $0 \leqslant x \leqslant a$ and $0 \leqslant y \leqslant b$

$$
\begin{aligned}
& \frac{\partial^{2}}{\partial x^{2}}+\frac{1}{2}(1+\nu) \frac{\partial^{2} v}{\partial x \partial y}+\frac{1}{2}(1-\nu) \frac{\partial^{2}}{\partial y^{2}}+\frac{\partial w}{\partial x} \frac{\partial^{2} w}{\partial x^{2}}+\frac{1}{2}(1+\nu) \frac{\partial w}{\partial y} \frac{\partial^{2} w}{\partial x \partial y} \\
& +\frac{1}{2}(1-\nu) \frac{\partial w}{\partial x} \frac{\partial^{2} w}{\partial y^{2}}=\frac{1-\nu^{2}}{E h}\left(\rho h \frac{\partial^{2}}{\partial t^{2}}+\frac{\partial N^{T}}{\partial x}\right) \\
& \frac{\partial^{2} v}{\partial y^{2}}+\frac{1}{2}(1+\nu) \frac{\partial^{2}}{\partial x \partial y}+\frac{1}{2}(1-\nu) \frac{\partial^{2} v}{\partial x^{2}}+\frac{\partial w}{\partial y} \frac{\partial^{2} w}{\partial y^{2}}+\frac{1}{2}(1+\nu) \frac{\partial w}{\partial x} \frac{\partial^{2} w}{\partial x \partial y} \\
& +\frac{1}{2}(1-\nu) \frac{\partial w}{\partial y} \frac{\partial^{2} w}{\partial x^{2}}=\frac{1-\nu^{2}}{E h}\left(\rho h \frac{\partial^{2} v}{\partial t^{2}}+\frac{\partial N^{T}}{\partial y}\right) \\
& D \nabla^{4} w+\rho h \frac{\partial^{2} w}{\partial t^{2}}+N^{T} \nabla^{2} w=\frac{1}{12} \rho h^{3} \frac{\partial^{2}}{\partial t^{2}}\left(\nabla^{2} w\right)-\nabla^{2} M^{T} \\
& +\frac{E h}{1-\nu^{2}}\left\{\left[\frac{\partial u}{\partial x}+\frac{1}{2}\left(\frac{\partial w}{\partial x}\right)^{2}+\nu\left(\frac{\partial v}{\partial y}+\frac{1}{2}\left(\frac{\partial w}{\partial y}\right)^{2}\right)\right] \frac{\partial^{2} w}{\partial x^{2}}\right. \\
& +(1-\nu)\left(\frac{\partial u}{\partial y}+\frac{\partial v}{\partial x}+\frac{\partial w}{\partial x} \frac{\partial w}{\partial y}\right) \frac{\partial^{2} w}{\partial x \partial y} \\
& \left.\quad+\left[\frac{\partial v}{\partial y}+\frac{1}{2}\left(\frac{\partial w}{\partial y}\right)^{2}+\nu\left(\frac{\partial u}{\partial x}+\frac{1}{2}\left(\frac{\partial w}{\partial x}\right)^{2}\right)\right] \frac{\partial^{2} w}{\partial y^{2}}\right\}+\frac{\varepsilon_{0} V_{p}^{2}}{2(d-w)^{2}}
\end{aligned}
$$

where 


$$
\begin{array}{ll}
M^{T}=\frac{E \alpha_{T}}{1-\nu} \int_{-h / 2}^{h / 2} z \theta d z & N^{T}=\frac{E \alpha_{T}}{1-\nu} \int_{-h / 2}^{h / 2} \theta d z \\
D=\frac{E h^{3}}{12\left(1-\nu^{2}\right.} & \nabla^{2}=\frac{\partial^{2}}{\partial x^{2}}+\frac{\partial^{2}}{\partial y^{2}}
\end{array}
$$

which are the thermal moment, thermal axial force, plate flexural rigidity and two-dimensional Laplacian operator, respectively, and $\theta=T-T_{0}$, in which $T(x, z, t)$ and $T_{0}$ are defined as the temperature field of the beam, and stress-free temperature (in equilibrium), respectively. Also, $t, \alpha_{T}, E, \nu, h, d, \varepsilon_{0}$ and $\rho$ are time, coefficient of thermal expansion, Young's modulus, Poisson's ratio, thickness of the microplate, capacitor gap, dielectric constant and material density, respectively. $u, v$ and $w$ are displacement components along with the $x, y$ and $z$ directions, respectively.

The thermal conduction equation, containing the thermoelastic coupling term, can be written as (Sun and Saka, 2009; Nayfeh and Pai, 2004)

$$
\kappa \nabla^{2} \theta+\kappa \frac{\partial^{2} \theta}{\partial z^{2}}=\rho c_{v} \frac{\partial \theta}{\partial t}+\beta T_{0} z \frac{\partial}{\partial t}\left(\frac{\partial u}{\partial x}+\frac{\partial v}{\partial y}-z\left(\frac{\partial^{2} w}{\partial x^{2}}+\frac{\partial^{2} w}{\partial y^{2}}\right)\right)
$$

where $c_{v}$ and $\kappa$ are the specific heat at constant volume and the thermal conductivity, respectively. $\beta=E \alpha_{T} /(1-2 \nu)$ is the thermal modulus.

So equations (2.1) and (2.3) represent the governing equations of nonlinear vibration of the micro-plate with thermoelastic damping (TED). In addition, thermal and elastic properties are assumed independent of temperature, and the temperature change due to TED is assumed to be small, thus the vibration of the micro-plate is investigated in a constant temperature $T_{0}$.

For convenience, the following nondimensional variables are introduced (denoted by hats)

$$
\begin{array}{llll}
\widehat{x}=\frac{x}{a} & \widehat{y}=\frac{y}{b} & \widehat{z}=\frac{z}{h} & \widehat{w}=\frac{w}{d} \\
\widehat{u}=\frac{u}{\bar{u}} & \widehat{v}=\frac{v}{\bar{v}} & \widehat{t}=\frac{t}{\bar{t}} & \widehat{\theta}=\frac{\theta}{\bar{\theta}}
\end{array}
$$

where

$$
\bar{u}=\frac{d^{2}}{a} \quad \bar{v}=\frac{d^{2}}{b} \quad \bar{t}=\frac{2 a b}{h} \sqrt{\frac{3 \rho}{E}} \quad \bar{\theta}=\frac{\beta T_{0} h^{2} d^{2}}{\bar{t} \kappa b^{2}}
$$

Substituting equations (2.4) into equations (2.1), the following equations are obtained

$$
\begin{aligned}
& \alpha_{1} \frac{\partial^{2} \widehat{u}}{\partial \widehat{x}^{2}}+\frac{1}{2}(1+\nu) \frac{\partial^{2} \widehat{v}}{\partial \widehat{x} \partial \widehat{y}}+\frac{1}{2}(1-\nu) \frac{\partial^{2} \widehat{u}}{\partial \widehat{y}^{2}}+\alpha_{1} \frac{\partial \widehat{w}}{\partial \widehat{x}} \frac{\partial^{2} \widehat{w}}{\partial \widehat{x}^{2}}+\frac{1}{2}(1+\nu) \frac{\partial \widehat{w}}{\partial \widehat{y}} \frac{\partial^{2} \widehat{w}}{\partial \widehat{x} \partial \widehat{y}} \\
&+ \frac{1}{2}(1-\nu) \frac{\partial \widehat{w}}{\partial \widehat{x}} \frac{\partial^{2} \widehat{w}}{\partial \widehat{y}^{2}}=\left(1-\nu^{2}\right) \alpha_{2} \frac{\partial^{2} \widehat{u}}{\partial \widehat{t}^{2}}+(1+\nu) \alpha_{3} \frac{\partial \widehat{N}^{T}}{\partial \widehat{x}} \\
& \alpha_{1}^{-1} \frac{\partial^{2} \widehat{v}}{\partial \widehat{y}^{2}}+\frac{1}{2}(1+\nu) \frac{\partial^{2} \widehat{u}}{\partial \widehat{x} \partial \widehat{y}}+\frac{1}{2}(1-\nu) \frac{\partial^{2} \widehat{v}}{\partial \widehat{x}^{2}}+\alpha_{1}^{-1} \frac{\partial \widehat{w}}{\partial \widehat{y}} \frac{\partial^{2} \widehat{w}}{\partial \widehat{y}^{2}}+\frac{1}{2}(1+\nu) \frac{\partial \widehat{w}}{\partial \widehat{x}} \frac{\partial^{2} \widehat{w}}{\partial \widehat{x} \partial \widehat{y}} \\
&+\frac{1}{2}(1-\nu) \frac{\partial \widehat{w}}{\partial \widehat{y}} \frac{\partial^{2} \widehat{w}}{\partial \widehat{x}^{2}}=\left(1-\nu^{2}\right) \alpha_{4} \frac{\partial^{2} \widehat{v}}{\partial \widehat{t}^{2}}+(1+\nu) \alpha_{5} \frac{\partial \widehat{N}^{T}}{\partial \widehat{y}}
\end{aligned}
$$




$$
\begin{aligned}
\alpha_{1} & \frac{\partial^{4} \widehat{w}}{\partial \widehat{x}^{4}}+2 \frac{\partial^{4} \widehat{w}}{\partial \widehat{x}^{2} \partial \widehat{y}^{2}}+\alpha_{1}^{-1} \frac{\partial^{4} \widehat{w}}{\partial \widehat{y}^{4}}+\left(1-\nu^{2}\right) \frac{\partial^{2} \widehat{w}}{\partial \widehat{t}^{2}}+(1+\nu) \widehat{N}^{T}\left(\alpha_{6} \frac{\partial^{2} \widehat{w}}{\partial \widehat{x}^{2}}+\alpha_{7} \frac{\partial^{2} \widehat{w}}{\partial \widehat{y}^{2}}\right) \\
= & \left(1-\nu^{2}\right) \frac{\partial^{2}}{\partial \widehat{t}^{2}}\left(\alpha_{2} \frac{\partial^{2} \widehat{w}}{\partial \widehat{x}^{2}}+\alpha_{4} \frac{\partial^{2} \widehat{w}}{\partial \widehat{y}^{2}}\right)-(1+\nu)\left(\alpha_{8} \frac{\partial^{2} \widehat{M}^{T}}{\partial \widehat{x}^{2}}+\alpha_{9} \frac{\partial^{2} \widehat{M}^{T}}{\partial \widehat{y}^{2}}\right) \\
& +12 \alpha_{10}^{2}\left\{\left[\alpha_{1} \frac{\partial \widehat{u}}{\partial \widehat{x}}+\frac{\alpha_{1}}{2}\left(\frac{\partial \widehat{w}}{\partial \widehat{x}}\right)^{2}+\nu\left(\frac{\partial \widehat{v}}{\partial \widehat{y}}+\frac{1}{2}\left(\frac{\partial \widehat{w}}{\partial \widehat{y}}\right)^{2}\right)\right] \frac{\partial^{2} \widehat{w}}{\partial \widehat{x}^{2}}\right. \\
& \left.+(1-\nu)\left(\frac{\partial \widehat{u}}{\partial \widehat{y}}+\frac{\partial \widehat{v}}{\partial \widehat{x}}+\frac{\partial \widehat{w}}{\partial \widehat{x}} \frac{\partial \widehat{w}}{\partial \widehat{y}}\right) \frac{\partial^{2} \widehat{w}}{\partial \widehat{x} \partial \widehat{y}}\right] \\
& \left.+\left[\alpha_{1}^{-1} \frac{\partial \widehat{v}}{\partial \widehat{y}}+\frac{1}{2} \alpha_{1}^{-1}\left(\frac{\partial \widehat{w}}{\partial \widehat{y}}\right)^{2}+\nu\left(\frac{\partial \widehat{u}}{\partial \widehat{x}}+\frac{1}{2}\left(\frac{\partial \widehat{w}}{\partial \widehat{x}}\right)^{2}\right)\right] \frac{\partial^{2} \widehat{w}}{\partial \widehat{y}^{2}}\right\}+\alpha_{11} \frac{V_{p}^{2}}{(1-\widehat{w})^{2}}
\end{aligned}
$$

In this model, Lifshitz and Roukes (2000) assumption is used. So the thermal gradient in the $z$ direction is much larger than the gradients in the $x$ and $y$ directions. Therefore, $\kappa \nabla^{2} \theta$ in equation (2.3) can be ignored. Thus the equation is simplified, and substituting equations (2.4) into equations $(2.3)$, yields

$$
\frac{\partial^{2} \widehat{\theta}}{\partial \widehat{z}^{2}}=\alpha_{12} \frac{\partial \widehat{\theta}}{\partial \widehat{t}}+\frac{\partial}{\partial \widehat{t}}\left(\alpha_{1} \frac{\partial \widehat{u}}{\partial \widehat{x}}+\frac{\partial \widehat{v}}{\partial \widehat{y}}-\frac{\widehat{z}}{\alpha_{10}}\left(\alpha_{1} \frac{\partial^{2} \widehat{w}}{\partial \widehat{x}^{2}}+\frac{\partial^{2} \widehat{w}}{\partial \widehat{y}^{2}}\right)\right)
$$

Equations (2.6) and (2.7) represent the nondimensional governing equations of the system, where

$$
\begin{array}{llll}
\alpha_{1}=\frac{b^{2}}{a^{2}} & \alpha_{2}=\frac{h^{2}}{12 a^{2}} & \alpha_{3}=\frac{\alpha_{T} \widehat{\theta} b^{2}}{d^{2}} & \alpha_{4}=\frac{h^{2}}{12 b^{2}} \\
\alpha_{5}=\frac{\alpha_{T} \widehat{\theta} a^{2}}{d^{2}} & \alpha_{6}=\frac{12 \alpha_{T} \widehat{\theta} b^{2}}{h^{2}} & \alpha_{7}=\frac{12 \alpha_{T} \widehat{\theta} a^{2}}{h^{2}} & \alpha_{8}=\frac{12 \alpha_{T} \widehat{\theta} b^{2}}{d h} \\
\alpha_{9}=\frac{12 \alpha_{T} \widehat{\theta} a^{2}}{d h} & \alpha_{10}=\frac{d}{h} & \alpha_{11}=\frac{\varepsilon_{0} a^{2} b^{2}}{2 d^{3} D} & \alpha_{12}=\frac{\rho c_{p} h^{3}}{2 a b \kappa} \sqrt{\frac{E}{3 \rho}}
\end{array}
$$

and

$$
\widehat{N}^{T}=\int_{-1 / 2}^{1 / 2} \widehat{\theta} d \widehat{z} \quad \widehat{M}^{T}=\int_{-1 / 2}^{1 / 2} \widehat{\theta} \widehat{z} d \widehat{z}
$$

It should be noted that the parameters of equations (2.8) and (2.9) are related as follows

$$
\frac{\alpha_{2}}{\alpha_{4}}=\alpha_{1} \quad \frac{\alpha_{8}}{\alpha_{9}}=\alpha_{2} \quad \alpha_{8}=\bar{\alpha} \alpha_{10} \alpha_{12} \quad \bar{\alpha}=\frac{12 \alpha_{T}^{2} E T_{0}}{(1-2 \nu) \rho c_{p}}
$$

\section{Large deformations under electrostatic load}

The microplate undergoes deflection under electrostatic voltage $V_{p}$. For calculating this deflection, the dynamic and thermoelastic terms should be eliminated form equations (2.6) 


$$
\begin{aligned}
& \alpha_{1} \frac{\partial^{2} \widehat{u}_{s}}{\partial \widehat{x}^{2}}+\frac{1}{2}(1+\nu) \frac{\partial^{2} \widehat{v}_{s}}{\partial \widehat{x} \partial \widehat{y}}+\frac{1}{2}(1-\nu) \frac{\partial^{2} \widehat{u}_{s}}{\partial \widehat{y}^{2}}+\alpha_{1} \frac{\partial \widehat{w}_{s}}{\partial \widehat{x}} \frac{\partial^{2} \widehat{w}_{s}}{\partial \widehat{x}^{2}}+\frac{1}{2}(1+\nu) \frac{\partial \widehat{w}_{s}}{\partial \widehat{y}} \frac{\partial^{2} \widehat{w}_{s}}{\partial \widehat{x} \partial \widehat{y}} \\
&+ \frac{1}{2}(1-\nu) \frac{\partial \widehat{w}_{s}}{\partial \widehat{x}} \frac{\partial^{2} \widehat{w}_{s}}{\partial \widehat{y}^{2}}=0 \\
& \alpha_{1}^{-1} \frac{\partial^{2} \widehat{v}_{s}}{\partial \widehat{y}^{2}}+\frac{1}{2}(1+\nu) \frac{\partial^{2} \widehat{u}_{s}}{\partial \widehat{x} \partial \widehat{y}}+\frac{1}{2}(1-\nu) \frac{\partial^{2} \widehat{v}_{s}}{\partial \widehat{x}^{2}}+\alpha_{1}^{-1} \frac{\partial \widehat{w}_{s}}{\partial \widehat{y}} \frac{\partial^{2} \widehat{w}_{s}}{\partial \widehat{y}^{2}} \\
&+\frac{1}{2}(1+\nu) \frac{\partial \widehat{w}_{s}}{\partial \widehat{x}} \frac{\partial^{2} \widehat{w}_{s}}{\partial \widehat{x} \partial \widehat{y}}+\frac{1}{2}(1-\nu) \frac{\partial \widehat{w}_{s}}{\partial \widehat{y}} \frac{\partial^{2} \widehat{w}_{s}}{\partial \widehat{x}^{2}}=0 \\
& \alpha_{1} \frac{\partial^{4} \widehat{w}_{s}}{\partial \widehat{x}^{4}}+2 \frac{\partial^{4} \widehat{w}_{s}}{\partial \widehat{x}^{2} \partial \widehat{y}^{2}}+\alpha_{1}^{-1} \frac{\partial^{4} \widehat{w}_{s}}{\partial \widehat{y}^{4}}=12 \alpha_{10}^{2}\left\{\left[\alpha \frac{\partial \widehat{u}_{s}}{\partial \widehat{x}}+\frac{\alpha_{1}}{2}\left(\frac{\partial \widehat{w}_{s}}{\partial \widehat{x}}\right)^{2}\right.\right. \\
&\left.+\nu\left(\frac{\partial \widehat{v}_{s}}{\partial \widehat{y}}+\frac{1}{2}\left(\frac{\partial \widehat{w}_{s}}{\partial \widehat{y}}\right)^{2}\right)\right] \frac{\partial^{2} \widehat{w}_{s}}{\partial \widehat{x}^{2}}+(1-\nu)\left(\frac{\partial \widehat{u}_{s}}{\partial \widehat{y}}+\frac{\partial \widehat{v}_{s}}{\partial \widehat{x}}+\frac{\partial \widehat{w}_{s}}{\partial \widehat{x}} \frac{\partial \widehat{w}_{s}}{\partial \widehat{y}}\right) \frac{\partial^{2} \widehat{w}_{s}}{\partial \widehat{x} \partial \widehat{y}} \\
&\left.+\left[\alpha_{1}^{-1} \frac{\partial \widehat{v}_{s}}{\partial \widehat{y}}+\frac{1}{2} \alpha_{1}^{-1}\left(\frac{\partial \widehat{w}_{s}}{\partial \widehat{y}}\right)^{2}+\nu\left(\frac{\partial \widehat{u}_{s}}{\partial \widehat{x}}+\frac{1}{2}\left(\frac{\partial \widehat{w}_{s}}{\partial \widehat{x}}\right)^{2}\right)\right] \frac{\partial^{2} \widehat{w}_{s}}{\partial \widehat{y}^{2}}\right\}+\alpha 11 \frac{V_{p}^{2}}{\left(1-\widehat{w}_{s}\right)^{2}}
\end{aligned}
$$

where $\widehat{u}_{s}, \widehat{v}_{s}$ and $\widehat{w}_{s}$ are static displacements. Nonlinear static equations (3.1) can be solved by Galerkin's method, using the following approximations (Szilared, 2004)

$$
\begin{aligned}
\widehat{w}_{s}(\widehat{x}, \widehat{y}) & =\sum_{m} \sum_{n} W_{m n}^{s} \varphi_{m n}(\widehat{x}, \widehat{y}) \quad \widehat{u}_{s}(\widehat{x}, \widehat{y})=\sum_{m} \sum_{n} U_{m n}^{s} \psi_{m n}(\widehat{x}, \widehat{y}) \\
\widehat{v}_{s}(\widehat{x}, \widehat{y}) & =\sum_{m} \sum_{n} V_{m n}^{s} \psi_{m n}(\widehat{x}, \widehat{y})
\end{aligned}
$$

To simplify the solution only the first term $(m=n=1)$ of equations (3.2) is considered. Substituting into equations (3.1) the functions $\varphi_{11}$ and $\psi_{11}$ as is listed in Table 1 with respect to the boundary conditions, equations (3.1) become

$$
\begin{aligned}
& \iint\left[\alpha_{1} U_{11}^{s} \frac{\partial^{2} \psi_{11}}{\partial \widehat{x}^{2}}+\frac{1}{2}(1+\nu) V_{11}^{s} \frac{\partial^{2} \psi_{11}}{\partial \widehat{x} \partial \widehat{y}}+\frac{1}{2}(1-\nu) U_{11}^{s} \frac{\partial^{2} \psi_{11}}{\partial \widehat{y}^{2}}\right. \\
& \quad+\alpha_{1}\left(W_{11}^{s} \frac{\partial \varphi_{11}}{\partial \widehat{x}}\right)\left(W_{11}^{s} \frac{\partial^{2} \varphi_{11}}{\partial \widehat{x}^{2}}\right)+\frac{1}{2}(1+\nu)\left(W_{11}^{s} \frac{\partial \varphi_{11}}{\partial \widehat{y}}\right)\left(W_{11}^{s} \frac{\partial^{2} \varphi_{11}}{\partial \widehat{x} \partial \widehat{y}}\right) \\
& \left.\quad+\frac{1}{2}(1-\nu)\left(W_{11}^{s} \frac{\partial \varphi_{11}}{\partial \widehat{x}}\right)\left(W_{11}^{s} \frac{\partial^{2} \varphi_{11}}{\partial \widehat{y}^{2}}\right)\right] \psi_{11} d \widehat{x} d \widehat{y}=0 \\
& \iint\left[\alpha_{1}^{-1} V_{11}^{s} \frac{\partial^{2} \psi_{11}}{\partial \widehat{y}^{2}}+\frac{1}{2}(1+\nu) U_{11}^{s} \frac{\partial^{2} \psi_{11}}{\partial \widehat{x} \partial \widehat{y}}+\frac{1}{2}(1-\nu) V_{11}^{s} \frac{\partial^{2} \psi_{11}}{\partial \widehat{x}^{2}}\right. \\
& \quad+\alpha_{1}^{-1}\left(W_{11}^{s} \frac{\partial \varphi_{11}}{\partial \widehat{y}}\right)\left(W_{11}^{s} \frac{\partial^{2} \varphi_{11}}{\partial \widehat{y}^{2}}\right)+\frac{1}{2}(1+\nu)\left(W_{11}^{s} \frac{\partial \varphi_{11}}{\partial \widehat{x}}\right)\left(W_{11}^{s} \frac{\partial^{2} \varphi_{11}}{\partial \widehat{x} \partial \widehat{y}}\right) \\
& \left.\quad+\frac{1}{2}(1-\nu)\left(W_{11}^{s} \frac{\partial \varphi_{11}}{\partial \widehat{x}}\right)\left(W_{11}^{s} \frac{\partial^{2} \varphi_{11}}{\partial \widehat{y}^{2}}\right)\right] \psi_{11} d \widehat{x} d \widehat{y}=0 \\
& \iint\left[\alpha_{1} W_{11}^{s} \frac{\partial^{4} \varphi_{11}}{\partial \widehat{x}^{4}}+2 W_{11}^{s} \frac{\partial^{4} \varphi_{11}}{\partial \widehat{x}^{2} \partial \widehat{y}^{2}}+\alpha_{1}^{-1} W_{11}^{s} \frac{\partial^{4} \varphi_{11}}{\partial \widehat{y}^{4}}-12 \alpha_{10}^{2}\left\{\left[\alpha_{1} U_{11}^{s} \frac{\partial \psi_{11}}{\partial \widehat{x}}\right.\right.\right. \\
& \left.\quad+\frac{\alpha_{1}}{2}\left(W_{11}^{s} \frac{\partial \varphi_{11}}{\partial \widehat{x}}\right)^{2}+\nu\left(V_{11}^{s} \frac{\partial \psi_{11}}{\partial \widehat{y}}+\frac{1}{2}\left(W_{11}^{s} \frac{\partial \varphi_{11}}{\partial \widehat{y}}\right)^{2}\right)\right] W_{11}^{s} \frac{\partial^{2} \varphi_{11}}{\partial \widehat{x}^{2}} \\
& \quad+(1-\nu)\left(U_{11}^{s} \frac{\partial \psi_{11}}{\partial \widehat{y}}+V_{11}^{s} \frac{\partial \psi_{11}}{\partial \widehat{x}}+W_{11}^{s} \frac{\partial \varphi_{11}}{\partial \widehat{x}} W_{11}^{s} \frac{\partial \varphi_{11}}{\partial \widehat{y}}\right) W_{11}^{s} \frac{\partial^{2} \varphi_{11}}{\partial \widehat{x} \partial \widehat{y}} \\
& \quad+\left[\alpha_{1}^{-1} V_{11}^{s} \frac{\partial \psi_{11}}{\partial \widehat{y}}+\frac{1}{2} \alpha_{1}^{-1}\left(W_{11}^{s} \frac{\partial \varphi_{11}}{\partial \widehat{y}}\right)^{2}\right. \\
& \left.\left.\left.\quad+\nu\left(U_{11}^{s} \frac{\partial \psi_{11}}{\partial \widehat{x}}+\frac{1}{2}\left(W_{11}^{s} \frac{\partial \varphi_{11}}{\partial \widehat{x}}\right)^{2}\right)\right] W_{11}^{s} \frac{\partial^{2} \varphi_{11}}{\partial \widehat{y}^{2}}\right\}-\frac{\alpha_{11} V_{p}^{2}}{\left(1-W_{11}^{s} \varphi_{11}\right)^{2}}\right] \varphi_{11} d \widehat{x} d \widehat{y}=0
\end{aligned}
$$


Table 1. List of functions $\psi_{11}$ and $\varphi_{11}$ with respect to the boundary condition (Leissa, 1969)

\begin{tabular}{|c|c|c|}
\hline 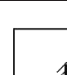 & \multirow{3}{*}{ 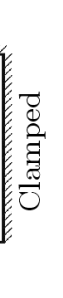 } & $C C C C$ \\
\hline 学 & & $\phi_{11}=[\cos (2 \pi \widehat{x})-1][\cos (2 \pi \widehat{y})-1]$ \\
\hline Clamped & & $\psi_{11}=\sin (\pi \widehat{x}) \sin (\pi \widehat{y})$ \\
\hline \multirow{3}{*}{ 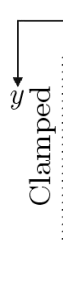 } & \multirow{3}{*}{ 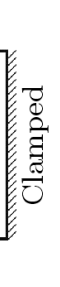 } & $C F C F$ \\
\hline & & $\phi_{11}=\cos (2 \pi \widehat{x})-1$ \\
\hline & & $\psi_{11}=\sin (\pi \widehat{x} \cos (\pi \widehat{y})$ \\
\hline
\end{tabular}

Introducing new parameters

$$
\begin{aligned}
A_{1} & =\iint\left[\alpha_{1} \frac{\partial^{2} \psi_{11}}{\partial \widehat{x}^{2}}+\frac{1}{2}(1-\nu) \frac{\partial^{2} \psi_{11}}{\partial \widehat{y}^{2}}\right] \psi_{11} d \widehat{x} d \widehat{y} \\
A_{2} & =\frac{1}{2}(1+\nu) \iint \frac{\partial^{2} \psi_{11}}{\partial \widehat{x} \partial \widehat{y}} \psi_{11} d \widehat{x} d \widehat{y} \\
A_{3} & =\iint\left[\alpha_{1} \frac{\partial \varphi_{11}}{\partial \widehat{x}} \frac{\partial^{2} \varphi_{11}}{\partial \widehat{x}^{2}}+\frac{1}{2}(1+\nu) \frac{\partial \varphi_{11}}{\partial \widehat{y}} \frac{\partial^{2} \varphi_{11}}{\partial \widehat{x} \partial \widehat{y}}+\frac{1}{2}(1-\nu) \frac{\partial \varphi_{11}}{\partial \widehat{x}} \frac{\partial^{2} \varphi_{11}}{\partial \widehat{y}^{2}}\right] \psi_{11} d \widehat{x} d \widehat{y} \\
A_{4} & =\iint\left[\alpha_{1}^{-1} \frac{\partial^{2} \psi_{11}}{\partial \widehat{y}^{2}}+\frac{1}{2}(1-\nu) \frac{\partial^{2} \psi_{11}}{\partial \widehat{x}^{2}}\right] \psi_{11} d \widehat{x} d \widehat{y} \\
A_{5} & =\iint\left[\alpha_{1}^{-1} \frac{\partial \varphi_{11}}{\partial \widehat{y}} \frac{\partial^{2} \varphi_{11}}{\partial \widehat{y}^{2}}+\frac{1}{2}(1+\nu) \frac{\partial \varphi_{11}}{\partial \widehat{x}} \frac{\partial^{2} \varphi_{11}}{\partial \widehat{x} \partial \widehat{y}}+\frac{1}{2}(1-\nu) \frac{\partial \varphi_{11}}{\partial \widehat{x}} \frac{\partial^{2} \varphi_{11}}{\partial \widehat{y}^{2}}\right] \psi_{11} d \widehat{x} d \widehat{y} \\
A_{6} & =\iint\left(\alpha_{1} \frac{\partial^{4} \varphi_{11}}{\partial \widehat{x}^{4}}+2 \frac{\partial^{4} \varphi_{11}}{\partial \widehat{x}^{2} \partial \widehat{y}^{2}}+\alpha_{1}^{-1} \frac{\partial^{4} \varphi_{11}}{\partial \widehat{y}^{4}}\right] \varphi_{11} d \widehat{x} d \widehat{y} \\
A_{7} & =-12 \alpha_{10}^{2} \iint\left[\alpha_{1} \frac{\partial \psi_{11}}{\partial \widehat{x}} \frac{\partial^{2} \varphi_{11}}{\partial \widehat{x}^{2}}+(1-\nu) \frac{\partial \psi_{11}}{\partial \widehat{y}} \frac{\partial^{2} \varphi_{11}}{\partial \widehat{x} \partial \widehat{y}}+\nu \frac{\partial \psi_{11}}{\partial \widehat{x}} \frac{\partial^{2} \varphi_{11}}{\partial \widehat{y}^{2}}\right] \varphi_{11} d \widehat{x} d \widehat{y} \\
A_{8} & =-12 \alpha_{10}^{2} \iint\left[\nu \frac{\partial \psi_{11}}{\partial \widehat{y}} \frac{\partial^{2} \varphi_{11}}{\partial \widehat{x}^{2}}+(1-\nu) \frac{\partial \psi_{11}}{\partial \widehat{x}} \frac{\partial^{2} \varphi_{11}}{\partial \widehat{x} \partial \widehat{y}}+\alpha_{1}^{-1} \frac{\partial \psi_{11}}{\partial \widehat{y}} W_{11}^{s} \frac{\partial^{2} \varphi_{11}}{\partial \widehat{y}^{2}}\right] \varphi_{11} d \widehat{x} d \widehat{y} \\
A_{9} & =-12 \alpha_{10}^{2} \iint\left\{\frac{\alpha_{1}}{2}\left(\frac{\partial \varphi_{11}}{\partial \widehat{x}}\right)^{2}+\frac{\nu}{2}\left(\frac{\partial \varphi_{11}}{\partial \widehat{y}}\right)^{2}\right] \frac{\partial^{2} \varphi_{11}}{\partial \widehat{x}^{2}}+(1-\nu) \frac{\partial \varphi_{11}}{\partial \widehat{x}} \frac{\partial \varphi_{11}}{\partial \widehat{y}} \frac{\partial^{2} \varphi_{11}}{\partial \widehat{x} \partial \widehat{y}} \\
& \left.+\left[\frac{1}{2} \alpha_{1}^{-1}\left(\frac{\partial \varphi_{11}}{\partial \widehat{y}}\right)^{2}+\frac{\nu}{2}\left(\frac{\partial \varphi_{11}}{\partial \widehat{x}}\right)^{2}\right] \frac{\partial^{2} \varphi_{11}}{\partial \widehat{y}^{2}}\right\} \varphi_{11} d \widehat{x} d \widehat{y} \\
F E & =\alpha_{11} V_{p}^{2} \iint \frac{\varphi_{11}}{\left(1-W_{11}^{s} \varphi_{11}\right)^{2}} d \widehat{x} d \widehat{y}
\end{aligned}
$$

Equations (3.3) can be rewritten as

$$
\begin{aligned}
& A_{1} U_{11}^{s}+A_{2} V_{11}^{s}+A_{3} W_{11}^{s}=0 \quad A_{2} U_{11}^{s}+A_{4} V_{11}^{s}+A_{5} W_{11}^{s}=0 \\
& A_{6} W_{11}^{s}+A_{7} U_{11}^{s} W_{11}^{s}+A_{8} V_{11}^{s} W_{11}^{s}+A_{9} W_{11}^{s}=0
\end{aligned}
$$

Now, by solving these equations, $U_{11}^{s}, V_{11}^{s}$ and $W_{11}^{s}$ can be calculated, and then substituting them into equations (3.2), the static deflection is obtained. 


\section{Transverse vibration around the static deflection}

The micro plate deflections have two components. The static deflections, as discussed in the previous section, due to the polarization voltage $V_{p}$ and the dynamic vibration deflections, occur around the static state. Consider only lateral vibration, thus the deflections $\widehat{u}(\widehat{x}, \widehat{y}), \widehat{v}(\widehat{x}, \widehat{y})$ and $\widehat{w}(\widehat{x}, \widehat{y}, \widehat{t})$ can be written as

$$
\begin{aligned}
& \widehat{w}(\widehat{x}, \widehat{y}, \widehat{t})=\widehat{w}_{s}(\widehat{x}, \widehat{y})+\widehat{w}_{d}(\widehat{x}, \widehat{y}, \widehat{t}) \quad \widehat{v}(\widehat{x}, \widehat{y}, \widehat{t})=\widehat{v}_{s}(\widehat{x}, \widehat{y}) \\
& \widehat{u}(\widehat{x}, \widehat{y}, \widehat{t})=\widehat{u}_{s}(\widehat{x}, \widehat{y})
\end{aligned}
$$

The equations describing vibration of the microplate around the static deflections are obtained by substituting equation (4.1) into equations $(2.6)_{3}$ and (2.7) and dropping the terms representing the equilibrium position that is the static deflection, equation $(3.1)_{3}$, and high order terms of the dynamic deflections. The result can be written as

$$
\begin{aligned}
\alpha_{1} & \frac{\partial^{4} \widehat{w}_{d}}{\partial \widehat{x}^{4}}+2 \frac{\partial^{4} \widehat{w}_{d}}{\partial \widehat{x}^{2} \partial \widehat{y}^{2}}+\alpha_{1}^{-1} \frac{\partial^{4} \widehat{w}_{d}}{\partial \widehat{y}^{4}}+\left(1-\nu^{2}\right) \frac{\partial^{2} \widehat{w}_{d}}{\partial \widehat{t}^{2}}+(1+\nu) \widehat{N}^{T}\left(\alpha_{6} \frac{\partial^{2} \widehat{w}_{s}}{\partial \widehat{x}^{2}}+\alpha_{7} \frac{\partial^{2} \widehat{w}_{s}}{\partial \widehat{y}^{2}}\right) \\
= & \left(1-\nu^{2}\right) \frac{\partial^{2}}{\partial \widehat{t}^{2}}\left(\alpha_{2} \frac{\partial^{2} \widehat{w}_{d}}{\partial \widehat{x}^{2}}+\alpha_{4} \frac{\partial^{2} \widehat{w}_{d}}{\partial \widehat{y}^{2}}\right)-(1+\nu)\left(\alpha_{8} \frac{\partial^{2} \widehat{M}^{T}}{\partial \widehat{x}^{2}}+\alpha_{9} \frac{\partial^{2} \widehat{M}^{T}}{\partial \widehat{y}^{2}}\right) \\
+ & 12 \alpha_{10}^{2}\left\{\left[\alpha_{1} \frac{\partial \widehat{u}_{s}}{\partial \widehat{x}}+\frac{\alpha_{1}}{2}\left(\frac{\partial \widehat{w}_{s}}{\partial \widehat{x}}\right)^{2}+\nu\left(\frac{\partial \widehat{v}_{s}}{\partial \widehat{y}}+\frac{1}{2}\left(\frac{\partial \widehat{w}_{s}}{\partial \widehat{y}}\right)^{2}\right)\right] \frac{\partial^{2} \widehat{w}_{d}}{\partial \widehat{x}^{2}}\right. \\
+ & {\left[\alpha_{1} \frac{\partial \widehat{w}_{s}}{\partial \widehat{x}} \frac{\partial \widehat{w}_{d}}{\partial \widehat{x}}+\nu\left(\frac{\partial \widehat{w}_{s}}{\partial \widehat{x}} \frac{\partial \widehat{w}_{d}}{\partial \widehat{x}}\right)\right] \frac{\partial^{2} \widehat{w}_{s}}{\partial \widehat{x}^{2}} } \\
+ & (1-\nu)\left(\frac{\partial \widehat{u}_{s}}{\partial \widehat{y}}+\frac{\partial \widehat{v}_{s}}{\partial \widehat{x}}+\frac{\partial \widehat{w}_{s}}{\partial \widehat{x}} \frac{\partial \widehat{w}_{s}}{\partial \widehat{y}}\right) \frac{\partial^{2} \widehat{w}_{d}}{\partial \widehat{x} \partial \widehat{y}}+(1-\nu)\left(\frac{\partial \widehat{w}_{s}}{\partial \widehat{x}} \frac{\partial \widehat{w}_{d}}{\partial \widehat{y}}+\frac{\partial \widehat{w}_{d}}{\partial \widehat{x}} \frac{\partial \widehat{w}_{s}}{\partial \widehat{y}}\right) \frac{\partial^{2} \widehat{w}_{s}}{\partial \widehat{x} \partial \widehat{y}} \\
+ & {\left[\alpha_{1}^{-1} \frac{\partial \widehat{v}_{s}}{\partial \widehat{y}}+\frac{1}{2} \alpha_{1}^{-1}\left(\frac{\partial \widehat{w}_{s}}{\partial \widehat{y}}\right)^{2}+\nu\left(\frac{\partial \widehat{u}_{s}}{\partial \widehat{x}}+\frac{1}{2}\left(\frac{\partial \widehat{w}_{s}}{\partial \widehat{x}}\right)^{2}\right)\right] \frac{\partial^{2} \widehat{w}_{d}}{\partial \widehat{y}^{2}} } \\
+ & {\left.\left[\alpha_{1}^{-1} \frac{\partial \widehat{w}_{s}}{\partial \widehat{y}} \frac{\partial \widehat{w}_{d}}{\partial \widehat{y}}+\nu\left(\frac{\partial \widehat{w}_{s}}{\partial \widehat{x}} \frac{\partial \widehat{w}_{d}}{\partial \widehat{x}}\right)\right] \frac{\partial^{2} \widehat{w}_{s}}{\partial \widehat{y}^{2}}\right\}+\frac{2 \alpha_{11} V_{p}^{2}}{\left(1-\widehat{w}_{s}\right)^{3}} \widehat{w}_{d}+\frac{2 \alpha_{11} V_{p}}{\left(1-\widehat{w}_{s}\right)^{2}} v(\widehat{t}) } \\
\frac{\partial^{2} \widehat{\theta}}{\partial \widehat{z}^{2}} & =\alpha_{12} \frac{\partial \widehat{\theta}}{\partial \widehat{t}}+\frac{\partial}{\partial \widehat{t}}\left(-\frac{\widehat{z}}{\alpha_{10}}\left(\alpha_{1} \frac{\partial^{2} \widehat{w}_{d}}{\partial \widehat{x}^{2}}+\frac{\partial^{2} \widehat{w}_{d}}{\partial \widehat{y}^{2}}\right)\right)
\end{aligned}
$$

For calculating the quality factor of TED, coupled thermoelastic equations (4.2) should be solved in the case of harmonic vibrations. So, $\widehat{w}_{d}$ and $\widehat{\theta}$ are set as in the following

$$
\begin{aligned}
& \widehat{w}_{d}(\widehat{x}, \widehat{y}, \widehat{z}, \widehat{t})=\sum_{n=0}^{\infty} \sum_{m=0}^{\infty} \bar{W}_{m n}(\widehat{x}, \widehat{y}, \widehat{z}) \mathrm{e}^{\mathrm{i} \Omega_{m n} \widehat{t}} \\
& \widehat{\theta}(\widehat{x}, \widehat{y}, \widehat{z}, \widehat{t})=\sum_{n=0}^{\infty} \sum_{m=0}^{\infty} \bar{\Theta}_{m n}(\widehat{x}, \widehat{y}, \widehat{z}) \mathrm{e}^{\mathrm{i} \Omega_{m n} \widehat{t}}
\end{aligned}
$$

where $\bar{W}_{m n}(\widehat{x}, \widehat{y})$ and $\bar{\Theta}_{m n}(\widehat{x}, \widehat{y}, \widehat{z})$ are the $(m, n)$-th transverse mode shapes of the plate, and the associated temperature variation, respectively, and $\Omega_{m n}$ is the complex frequency that has the real part $\omega_{m n}$, and the imaginary part $\lambda_{m n}$ which is related to the damping. Therefore, substituting equations (4.3) into the equation of transverse vibration around static deflection $(4.4)_{1}$ and thermal conduction equation $(4.4)_{2}$ yields 


$$
\begin{aligned}
& \alpha_{1} \frac{\partial^{4} \bar{W}_{m n}}{\partial \widehat{x}^{4}}+2 \frac{\partial^{4} \bar{W}_{m n}}{\partial \widehat{x}^{2} \partial \widehat{y}^{2}}+\alpha_{1}^{-1} \frac{\partial^{4} \bar{W}_{m n}}{\partial \widehat{y}^{4}}-\Omega_{m n}^{2}\left(1-\nu^{2}\right) \bar{W}_{m n} \\
& +(1+\nu) \widehat{N}_{m n}^{T}\left(\alpha_{6} \frac{\partial^{2} \widehat{w}_{s}}{\partial \widehat{x}^{2}}+\alpha_{7} \frac{\partial^{2} \widehat{w}_{s}}{\partial \widehat{y}^{2}}\right)=-\Omega_{m n}^{2}\left(1-\nu^{2}\right)\left(\alpha_{2} \frac{\partial^{2} \bar{W}_{m n}}{\partial \widehat{x}^{2}}+\alpha_{4} \frac{\partial^{2} \bar{W}_{m n}}{\partial \widehat{y}^{2}}\right) \\
& -(1+\nu)\left(\alpha_{8} \frac{\partial^{2} \widehat{M}_{m n}^{T}}{\partial \widehat{x}^{2}}+\alpha_{9} \frac{\partial^{2} \widehat{M}_{m n}^{T}}{\partial \widehat{y}^{2}}\right)+12 \alpha_{10}^{2}\left\{\left[\alpha_{1} \frac{\partial \widehat{u}_{s}}{\partial \widehat{x}}+\alpha_{\frac{1}{2}}\left(\frac{\partial \widehat{w}_{s}}{\partial \widehat{x}}\right)^{2}\right.\right. \\
& \left.+\nu\left(\frac{\partial \widehat{v}_{s}}{\partial \widehat{y}}+\frac{1}{2}\left(\frac{\partial \widehat{w}_{s}}{\partial \widehat{y}}\right)^{2}\right)\right] \frac{\partial^{2} \bar{W}_{m n}}{\partial \widehat{x}^{2}}+\left[\alpha_{1} \frac{\partial \widehat{w}_{s}}{\partial \widehat{x}} \frac{\partial \bar{W}_{m n}}{\partial \widehat{x}}+\nu\left(\frac{\partial \widehat{w}_{s}}{\partial \widehat{x}} \frac{\partial \bar{W}_{m n}}{\partial \widehat{x}}\right)\right] \frac{\partial^{2} \widehat{w}_{s}}{\partial \widehat{x}^{2}} \\
& +(1-\nu)\left(\frac{\partial \widehat{u}_{s}}{\partial \widehat{y}}+\frac{\partial \widehat{v}_{s}}{\partial \widehat{x}}+\frac{\partial \widehat{w}_{s}}{\partial \widehat{x}} \frac{\partial \widehat{w}_{s}}{\partial \widehat{y}}\right) \frac{\partial^{2} \bar{W}_{m n}}{\partial \widehat{x} \partial \widehat{y}} \\
& +(1-\nu)\left(\frac{\partial \widehat{w}_{s}}{\partial \widehat{x}} \frac{\partial \bar{W}_{m n}}{\partial \widehat{y}}+\frac{\partial \bar{W}_{m n}}{\partial \widehat{x}} \frac{\partial \widehat{w}_{s}}{\partial \widehat{y}}\right) \frac{\partial^{2} \widehat{w}_{s}}{\partial \widehat{x} \partial \widehat{y}} \\
& +\left[\alpha_{1}^{-1} \frac{\partial \widehat{v}_{s}}{\partial \widehat{y}}+\frac{1}{2} \alpha_{1}^{-1}\left(\frac{\partial \widehat{w}_{s}}{\partial \widehat{y}}\right)^{2}+\nu\left(\frac{\partial \widehat{u}_{s}}{\partial \widehat{x}}+\frac{1}{2}\left(\frac{\partial \widehat{w}_{s}}{\partial \widehat{x}}\right)^{2}\right)\right] \frac{\partial^{2} \bar{W}_{m n}}{\partial \widehat{y}^{2}} \\
& \left.+\left[\alpha_{1}^{-1} \frac{\partial \widehat{w}_{s}}{\partial \widehat{y}} \frac{\partial \bar{W}_{m n}}{\partial \widehat{y}}+\nu\left(\frac{\partial \widehat{w}_{s}}{\partial \widehat{x}} \frac{\partial \bar{W}_{m n}}{\partial \widehat{x}}\right)\right] \frac{\partial^{2} \widehat{w}_{s}}{\partial \widehat{y}^{2}}\right\}+\frac{2 \alpha_{11} V_{p}^{2}}{\left(1-\widehat{w}_{s}\right)^{3}} \bar{W}_{m n} \\
& \frac{\partial^{2} \bar{\Theta}_{m n}}{\partial \widehat{z}^{2}}=\alpha_{12} \frac{\partial \bar{\Theta}_{m n}}{\partial \widehat{t}}+\frac{\partial}{\partial \widehat{t}}\left(-\frac{\widehat{z}}{\alpha_{10}}\left(\alpha_{1} \frac{\partial^{2} \bar{W}_{m n}}{\partial \widehat{x}^{2}}+\frac{\partial^{2} \bar{W}_{m n}}{\partial \widehat{y}^{2}}\right)\right)
\end{aligned}
$$

where

$$
\widehat{N}_{m n}^{T}=\int_{-1 / 2}^{1 / 2} \Theta_{m n} d \widehat{z} \quad \widehat{M}_{m n}^{T}=\int_{-1 / 2}^{1 / 2} \Theta_{m n} \widehat{z} d \widehat{z}
$$

Assuming that there is no heat flow across the upper and lower surface of the beam, the boundary conditions for solving equation $(4.4)_{2}$ are $\partial \Theta_{m n} / \partial \widehat{z}=0$ at $\widehat{z}= \pm 1 / 2$. Then solving equation $(4.4)_{2}$, and substituting the results into equation (4.5), the following equations are obtained

$$
\widehat{M}_{m n}^{T}=\frac{1}{\alpha_{10} \alpha_{12}} C_{m n}^{T}\left(\alpha_{1} \frac{\partial^{2} W_{m n}}{\partial \widehat{x}^{2}}+\frac{\partial^{2} W_{m n}}{\partial \widehat{y}^{2}}\right) \quad \widehat{N}_{m n}^{T}=0
$$

where

$$
C_{m n}^{T}=\frac{1}{12}-\frac{2}{N_{m n}^{3}}\left[\tan \left(\frac{N_{m n}}{2}\right)-\frac{N_{m n}}{2}\right] \quad N_{m n}=(1-i) \sqrt{\frac{\Omega_{m n} \alpha_{12}}{2}}
$$

Therefore, equations (4.6) should be substituted into equation $(4.4)_{1}$. Since in the case of free vibration the amplitude of higher harmonic terms are dramatically small relative to the primary amplitude, their effects on thermoelastic damping are negligible. Thus by considering $m=n=1$ and setting $\bar{W}_{m n}=\varphi_{m n}$, Galerkin's method is used for calculating $\Omega_{m n}$, see Hagedorn and Gupta (2007). The function $\varphi_{11}$ should satisfy the boundary conditions and is selected with respect to the boundary conditions that are listed in Table 1 . Therefore, equation $(4.4)_{1}$ should be rewritten as 


$$
\begin{aligned}
\iint & {\left[\alpha_{1} \frac{\partial^{4} \varphi_{11}}{\partial \widehat{x}^{4}}+2 \frac{\partial^{4} \varphi_{11}}{\partial \widehat{x}^{2} \partial \widehat{y}^{2}}+\alpha_{1}^{-1} \frac{\partial^{4} \varphi_{11}}{\partial \widehat{y}^{4}}-\Omega_{m n}^{2}\left(1-\nu^{2}\right) \varphi_{11}+\Omega_{m n}^{2}\left(1-\nu^{2}\right)\left(\alpha_{2} \frac{\partial^{2} \varphi_{11}}{\partial \widehat{x}^{2}}\right.\right.} \\
& \left.+\alpha_{4} \frac{\partial^{2} \varphi_{11}}{\partial \widehat{y}^{2}}\right)+(1+\nu) \bar{\alpha} C_{m n}^{T}\left(\alpha_{1} \frac{\partial^{4} \varphi_{11}}{\partial \widehat{x}^{4}}+2 \frac{\partial^{4} \varphi_{11}}{\partial \widehat{x}^{2} \partial \widehat{y}^{2}}+\alpha_{1}^{-1} \frac{\partial^{4} \varphi_{11}}{\partial \widehat{y}^{4}}\right) \\
& -12 \alpha_{10}^{2}\left\{\left[\alpha_{1} \frac{\partial \widehat{u}_{s}}{\partial \widehat{x}}+\frac{\alpha_{1}}{2}\left(\frac{\partial \widehat{w}_{s}}{\partial \widehat{x}}\right)^{2}+\nu\left(\frac{\partial \widehat{v}_{s}}{\partial \widehat{y}}+\frac{1}{2}\left(\frac{\partial \widehat{w}_{s}}{\partial \widehat{y}}\right)^{2}\right)\right] \frac{\partial^{2} \varphi_{11}}{\partial \widehat{x}^{2}}\right. \\
& +\left[\alpha_{1} \frac{\partial \psi_{11}}{\partial \widehat{x}}+\alpha_{1} \frac{\partial \widehat{w}_{s}}{\partial \widehat{x}} \frac{\partial \varphi_{11}}{\partial \widehat{x}}+\nu\left(\frac{\partial \psi_{11}}{\partial \widehat{y}}+\frac{\partial \widehat{w}_{s}}{\partial \widehat{x}} \frac{\partial \varphi_{11}}{\partial \widehat{x}}\right)\right] \frac{\partial^{2} \widehat{w}_{s}}{\partial \widehat{x}^{2}} \\
& +(1-\nu)\left(\frac{\partial \widehat{u}_{s}}{\partial \widehat{y}}+\frac{\partial \widehat{v}_{s}}{\partial \widehat{x}}+\frac{\partial \widehat{w}_{s}}{\partial \widehat{x}} \frac{\partial \widehat{w}_{s}}{\partial \widehat{y}}\right) \frac{\partial^{2} \varphi_{11}}{\partial \widehat{x} \partial \widehat{y}} \\
& +(1-\nu)\left(\frac{\partial \psi_{11}}{\partial \widehat{y}}+\frac{\partial \psi_{11}}{\partial \widehat{x}}+\frac{\partial \widehat{w}_{s}}{\partial \widehat{x}} \frac{\partial \varphi_{11}}{\partial \widehat{y}}+\frac{\partial \varphi_{11}}{\partial \widehat{x}} \frac{\partial \widehat{w}_{s}}{\partial \widehat{y}}\right) \frac{\partial^{2} \widehat{w}_{s}}{\partial \widehat{x} \partial \widehat{y}} \\
& +\left[\alpha_{1}^{-1} \frac{\partial \widehat{v}_{s}}{\partial \widehat{y}}+\frac{1}{2} \alpha_{1}^{-1}\left(\frac{\partial \widehat{w}_{s}}{\partial \widehat{y}}\right)^{2}+\nu\left(\frac{\partial \widehat{u}_{s}}{\partial \widehat{x}}+\frac{1}{2}\left(\frac{\partial \widehat{w}_{s}}{\partial \widehat{x}}\right)^{2}\right)\right] \frac{\partial^{2} \varphi_{11}}{\partial \widehat{y}^{2}}+\left[\alpha_{1}^{-1} \frac{\partial \psi_{11}}{\partial \widehat{y}}\right. \\
& \left.\left.\left.+\alpha_{1}^{-1} \frac{\partial \widehat{w}_{s}}{\partial \widehat{y}} \frac{\partial \varphi_{11}}{\partial \widehat{y}}+\nu\left(\frac{\partial \psi_{11}}{\partial \widehat{x}}+\frac{\partial \widehat{w}_{s}}{\partial \widehat{x}} \frac{\partial \varphi_{11}}{\partial \widehat{x}}\right)\right] \frac{\partial^{2} \widehat{w}_{s}}{\partial \widehat{y}^{2}}\right\}-\frac{2 \alpha_{11} V_{p}^{2}}{\left(1-\widehat{w}_{s}\right)^{3}} \varphi_{11}\right] \varphi_{11} d \widehat{x} d \widehat{y}
\end{aligned}
$$

Introducing new parameters

$$
\begin{aligned}
L_{1} & =\alpha_{1}\left[1+(1+\nu) \bar{\alpha} C_{11}^{T}\right] \quad L_{2}=2\left[1+(1+\nu) \bar{\alpha} C_{11}^{T}\right] \\
L_{3} & =\alpha_{1}^{-1}\left[1+(1+\nu) \bar{\alpha} C_{11}^{T}\right] \quad P_{1}=\iint \frac{\partial^{4} \varphi_{11}}{\partial \widehat{x}^{4}} \varphi_{11} d \widehat{x} d \widehat{y} \\
P_{2} & =\iint \frac{\partial^{4} \varphi_{11}}{\partial \widehat{x}^{2} \partial \widehat{y}^{2}} \varphi_{11} d \widehat{x} d \widehat{y} \quad P_{3}=\iint \frac{\partial^{4} \varphi_{11}}{\partial \widehat{y}^{4}} \varphi_{11} d \widehat{x} d \widehat{y} \\
P_{4} & =\iint \varphi_{11}^{2} d \widehat{x} d \widehat{y} \quad P_{5}=\left(1-\nu^{2}\right) \iint\left[\left(\alpha_{2} \frac{\partial^{2} \varphi_{11}}{\partial \widehat{x}^{2}}+\frac{\alpha_{2}}{\alpha_{1}} \frac{\partial^{2} \varphi_{11}}{\partial \widehat{y}^{2}}\right)-\varphi_{11}\right] \varphi_{11} d \widehat{x} d \widehat{y} \\
P_{6} & =\iint\left\{-12 \alpha_{10}^{2}\left\{\left[\alpha_{1} \frac{\partial \widehat{u}_{s}}{\partial \widehat{x}}+\frac{\alpha_{1}}{2}\left(\frac{\partial \widehat{w}_{s}}{\partial \widehat{x}}\right)^{2}+\nu\left(\frac{\partial \widehat{v}_{s}}{\partial \widehat{y}}+\frac{1}{2}\left(\frac{\partial \widehat{w}_{s}}{\partial \widehat{y}}\right)^{2}\right)\right] \frac{\partial^{2} \varphi_{11}}{\partial \widehat{x}^{2}}\right.\right. \\
& +\left[\alpha_{1} \frac{\partial \widehat{w}_{s}}{\partial \widehat{x}} \frac{\partial \varphi_{11}}{\partial \widehat{x}}+\nu\left(\frac{\partial \widehat{w}_{s}}{\partial \widehat{x}} \frac{\partial \varphi_{11}}{\partial \widehat{x}}\right)\right] \frac{\partial^{2} \widehat{w}_{s}}{\partial \widehat{x}^{2}}+(1-\nu)\left(\frac{\partial \widehat{u}_{s}}{\partial \widehat{y}}+\frac{\partial \widehat{v}_{s}}{\partial \widehat{x}}+\frac{\partial \widehat{w}_{s}}{\partial \widehat{x}} \frac{\partial \widehat{w}_{s}}{\partial \widehat{y}}\right) \frac{\partial^{2} \varphi_{11}}{\partial \widehat{x} \partial \widehat{y}} \\
& +(1-\nu)\left(\frac{\partial \widehat{w}_{s}}{\partial \widehat{x}} \frac{\partial \varphi_{11}}{\partial \widehat{y}}+\frac{\partial \varphi_{11}}{\partial \widehat{x}} \frac{\partial \widehat{w}_{s}}{\partial \widehat{y}}\right) \frac{\partial^{2} \widehat{w}_{s}}{\partial \widehat{x} \partial \widehat{y}} \\
& +\left[\alpha_{1}^{-1} \frac{\partial \widehat{v}_{s}}{\partial \widehat{y}}+\frac{1}{2} \alpha_{1}^{-1}\left(\frac{\partial \widehat{w}_{s}}{\partial \widehat{y}}\right)^{2}+\nu\left(\frac{\partial \widehat{u}_{s}}{\partial \widehat{x}}+\frac{1}{2}\left(\frac{\partial \widehat{w}_{s}}{\partial \widehat{x}}\right)^{2}\right)\right] \frac{\partial^{2} \varphi_{11}}{\partial \widehat{y}^{2}} \\
& \left.\left.+\left[\alpha_{1}^{-1} \frac{\partial \widehat{w}_{s}}{\partial \widehat{y}} \frac{\partial \varphi_{11}}{\partial \widehat{y}}+\nu\left(\frac{\partial \widehat{w}_{s}}{\partial \widehat{x}} \frac{\partial \varphi_{11}}{\partial \widehat{x}}\right)\right] \frac{\partial^{2} \widehat{w}_{s}}{\partial \widehat{y}^{2}}\right\}-\frac{2 \alpha_{11} V_{p}^{2}}{\left(1-\widehat{w}_{s}\right)^{3}} \varphi_{11}\right\} \varphi_{11} d \widehat{x} d \widehat{y}
\end{aligned}
$$

Equation (4.8) can be written as follows

$$
L_{1} P_{1}+L_{2} P_{2}+L_{3} P_{3}+\Omega_{11}^{2} P_{5}+P_{6}=0
$$

Finally, by calculating $\Omega_{11}$ from equation (4.10) and separating the real and imaginary parts, the quality factor of TED for large deformation of the microplate is obtained

$$
Q^{-1}=2\left|\frac{\lambda_{11}}{\omega_{11}}\right|
$$




\section{Pull-in voltage}

Beyond the maximum value of DC voltage, the microplate of the resonator snaps and touches the rigid plate. This maximum value, denoted by $V_{M}$, is called pull-in voltage (Batra et al., 2007). For calculating $V_{M}$, the minimum voltage at which the microplate becomes unstable, should be found. For example, for a microplate whose properties are listed in Table 2, the pull-in voltage is shown in Fig. 1. As can be seen in this figure, beyond this voltage, that the microplate becomes unstable and the deflection grows suddenly. In this case, the pull-in voltage is $V_{M}=130.73 \mathrm{~V}$ and the related deflection is $\widehat{w}_{\max }=0.45$.

Table 2. List of geometrical and material properties of the microplate

\begin{tabular}{|c|c|c|c|c|c|}
\hline$h[\mu \mathrm{m}]$ & $d[\mu \mathrm{m}]$ & $a[\mu \mathrm{m}]$ & $b[\mu \mathrm{m}]$ & $T_{0}[\mathrm{~K}]$ & $\kappa\left[\mathrm{Wm}^{-1} \mathrm{~K}^{-1}\right]$ \\
\hline \hline 1.5 & 1.2 & 200 & 100 & 300 & 148 \\
\hline$c_{p}\left[\mathrm{Jkg}^{-1} \mathrm{~K}^{-1}\right]$ & $\alpha_{T}\left[10^{-6} \mathrm{~K}^{-1}\right]$ & $E[\mathrm{Gpa}]$ & $v[-]$ & $\rho\left[\mathrm{kg} \mathrm{m}^{-3}\right]$ & $\varepsilon_{0}\left[\mathrm{C}^{2} \mathrm{~m}^{-2} \mathrm{~N}^{-1}\right]$ \\
\hline \hline 712 & 2.6 & 170 & 0.25 & 2330 & $8.85 \cdot 10^{-12}$ \\
\hline
\end{tabular}

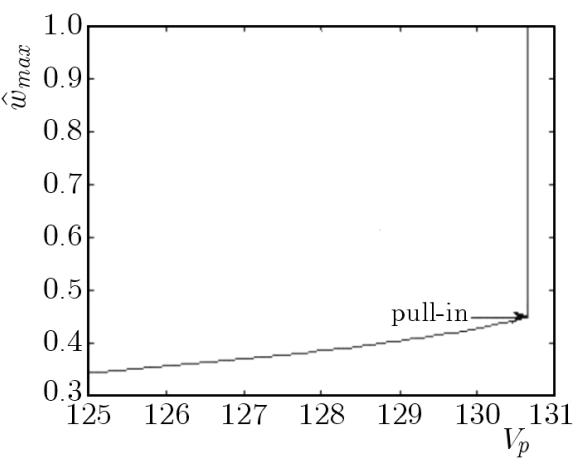

Fig. 1. Pull-in voltage in the microplate with properties listed in Table 2

\section{Results}

Francais and Dufour (1999) measured the centre deflection of a fully clamped square microplate under various electrostatic actuations. They depicted the center deflection versus the following parameter

$$
C D=\frac{\varepsilon_{0} V_{p}^{2}(a b)^{2}}{32 d^{3} D}
$$

In Fig. 2, the static deflection at the centre of the plate $\widehat{w}_{\max }$, which is calculated here using the Galerkin's method, is compared with the experimental results of Francais and Dufour (1999). There is a good agreement between Francais and Dufour's measurments and the large deformation model of microplate.

In Fig. 3, the large deformation model in two cases $\nu=0$ and $\nu=0.25$ are compared with Lifshitz model (Lifshitz and Roukes, 2000) in which the TED is calculated for the clampedclamped microbeam. The microplate and microbeam have the same properties that are listed in Table 2. The microplate is also considered with the CFCF boundary condition and without electrical load $\left(V_{p}=0\right)$. As can be seen in Fig. 3, TED in the Lifshitz model and the microplate with $\nu=0$ are in good agreement.

In Fig. 4, the TED of large deformation model is depicted versus $\bar{\alpha}$ based on the configuration of Table 2, for two cases of voltages: $V_{p}=0.1 V_{M}$ and $V_{p}=0.9 V_{M} \cdot \bar{\alpha}$ is an important parameter 


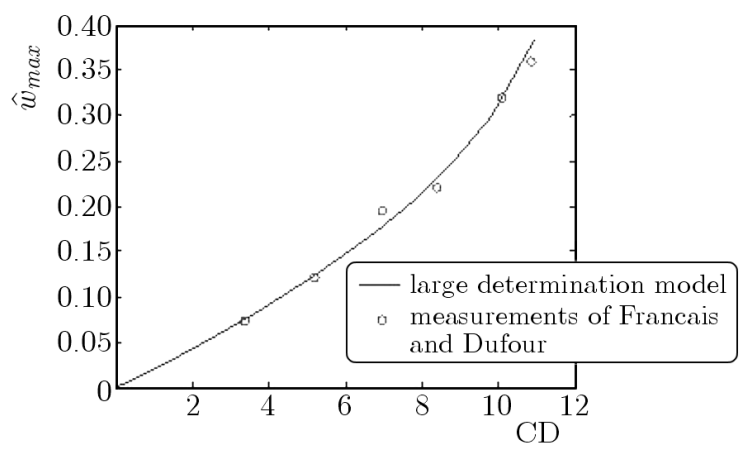

Fig. 2. Comparison of $\widehat{w}_{\max }$ calculated using Galerkin's method with the experimental results of Francais and Dufour (1999)

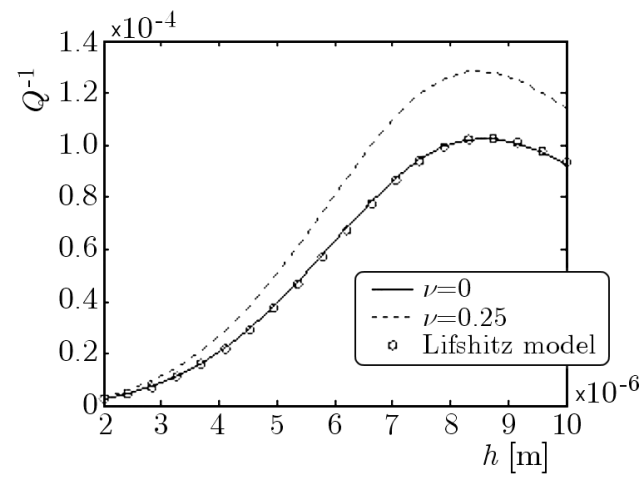

Fig. 3. Comparison of TED in large deformation models of the microplate $(\nu=0$ and $\nu=0.25)$ with the Lifshitz model

because it represents the properties of the material. As can be seen in this figure, by increasing $\bar{\alpha}$ the difference between two cases becomes larger. In small values of $\bar{\alpha}$, these two cases are coincided, so small $\bar{\alpha}$ can change the nonlinear model to a linear model of the microplate. For example, $\bar{\alpha}$ of silicon is $\bar{\alpha}=0.005$, thus silicon has linear properties.

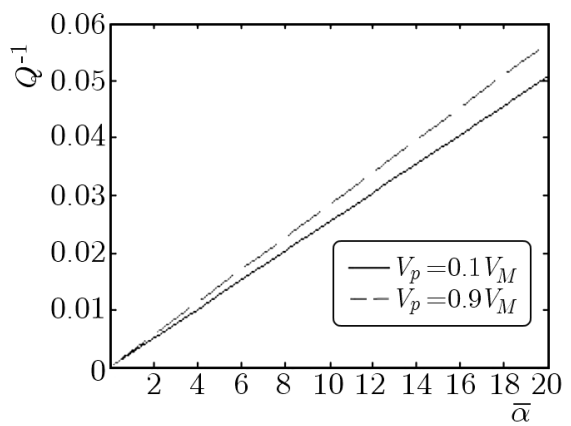

Fig. 4. TED of the large deformation model versus $\bar{\alpha}$

In Fig. 5, TED is depicted versus $\alpha_{1}$ that is geometrical parameter for $\bar{\alpha}=\alpha_{10}=\alpha_{11}=$ $\alpha_{12}=1, \alpha_{2}=0.1, V_{M}=10.0899$ and $\nu=0.25$. As can be seen in this figure, there is a critical value of $\alpha_{1}$ in which TED has the maximum value. Also, the maximum values of TED are increased by growing the electrostatic load. 


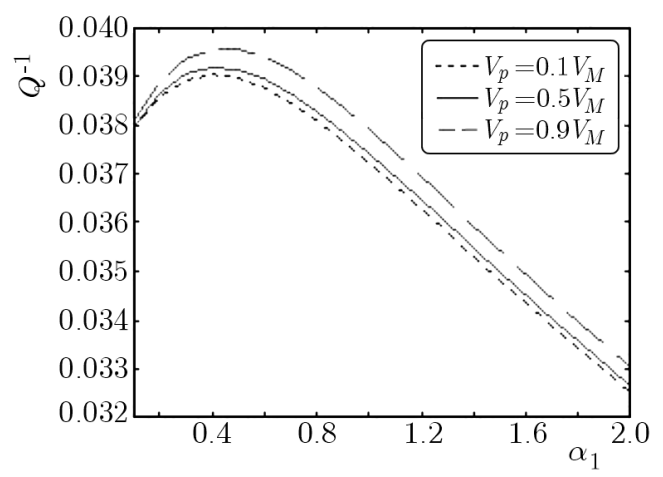

Fig. 5. TED of the large deformation model versus $\bar{\alpha}$

\section{Conclusion}

In this paper, a resonator is modeled as a rectangular microplate. TED of the microplate is calculated by linear and nonlinear assumptions. In the large deformation model, large deformation due to electrostatic load is considered by von-Karman assumptions. For calculating the thermoelastic damping, the static and vibration equations are solved by using Galerkin's method.

The material properties may exhibit nonlinear effects on TED, but silicon has linear properties. Figures 4 and 5 have useful results for MEMS resonator designers about material properties and geometrical dimensions of the resonators. In the future work, the thermoelastic damping of a thick plate can be studied, and the thermal conduction equation can be solved in two dimensional space.

\section{References}

1. Abdel-Rahman E.M., Younis M.I., Nayfeh A.H., 2002, Characterization of the mechanical behavior of an electrically actuated microbeam, Journal of Micromechanics and Microengineering, 12, 759-766

2. Alblas J.B., 1981, A note on the theory of thermoelastic damping, Journal of Thermal Stresses, 4, 3/4, 333-355

3. Batra R.C., Porfiri M., Spinello D., 2007, Review of modeling electrostatically actuated microelectromechanical systems, Smart Materials and Structures, 16, 6, R23-R31

4. Сног J., Сно М., Rнiм J., 2010, Efficient prediction of the quality factors of micromechanical resonators, Journal of Sound and Vibration, 329, 1, 84-95

5. Fargas M.A., Costa Castelló R., Shkel A.M., 2005, Modelling the electrostatic actuation of MEMS: state of the art 2005, Technical Report

6. Francais O., Dufour I., 1999, Normalized abacus for the global behavior of diaphragms: pneumatic, electrostatic, piezoelectric or electromagnetic actuation, Journal of Modeling and Simulation of Microsystems, 2, 149-160

7. Guo F.L., Rogerson G.A., 2003, Thermoelastic coupling effect on a micro-machined beam resonator, Mechanics Research Communications, 30, 6, 513-518

8. Hagedorn P., Gupta A.D., 2007, Vibrations and Waves in Continuous Mechanical Systems, Wiley

9. Leissa A.W., 1969, Vibration of Plates, DC: NASA, Washington

10. Lifshitz R., Roukes M.L., 2000, Thermoelastic damping in micro- and nanomechanical systems, Physical Review B, 61, 8, 5600-5609 
11. Nayfeh A.H., PAi P.F., 2004, Linear and Nonlinear Structural Mechanics, Wiley, New Jersey

12. NAYFeh A.H., Younis M.I., 2004a, A new approach to the modeling and simulation of flexible microstructures under the effect of squeeze-film damping, Journal of Micromechanics and Microengineering, 14, 2, 170-181

13. NAYFeh A.H., Younis M.I., 2004b, Modeling and simulations of thermoelastic damping in microplates, Journal of Micromechanics and Microengineering, 14, 1711-1717

14. Prabhakar S., Vengallatore S., 2008, Theory of thermoelastic damping in micromechanical resonators with two-dimensional heat conduction, Journal of Microelectromechanical Systems, 17, 2, 494-502

15. Serra E., Bonaldi M., 2009, A finite element formulation for thermoelastic damping analysis, International Journal for Numerical Methods in Engineering, 78, 6, 671-691

16. Sudipto K. De, Aluru N.R., 2006, Theory of thermoelastic damping in electrostatically actuated microstructures, Physical Review B, 10, 144305-144318

17. Sun Y., SAKa M., 2009, Thermoelastic damping in micro-scale circular plate resonators, Journal of Sound and Vibration, 310, 392-405

18. Yi Y.-B., Matin M.A., 2007, Eigenvalue solution of thermoelastic damping in beam resonators using a finite element analysis, Journal of Vibration and Acoustics, 129, 4, 478-483

19. Zener C., 1937, Internal friction in solids. I. Theory of internal friction in reeds, Physical Review, 52,3, 230-235

20. Zener C., 1938, Internal friction in solids. II. General theory of thermoelastic internal friction, Physical Review, 53, 90-99

Manuscript received March 17, 2013; accepted for print September 27, 2014 